\title{
Additive Approximation for Layer Minimization of Manhattan Switchbox Routing
}

\author{
Dávid Szeszlér ${ }^{1,2}$ \\ Department of Computer Science and Information Theory \\ Budapest University of Technology and Economics \\ Budapest, Hungary
}

\begin{abstract}
Switchbox routing is one of the many problems arising in the field of VLSI routing. It requires interconnecting given sets of terminals that are placed on the boundaries of a rectangular circuit board using a 3-dimensional grid in a vertex-disjoint way.

An important special case is the Manhattan Switchbox Routing problem. Here minimizing the number of layers of a routing (that is, the height of the grid) is known to be NP-hard. In this paper we provide a linear time algorithm that solves any such problem on a number of layers that is greater by at most 5 than the optimum.
\end{abstract}

Keywords: VLSI, Switchbox Routing, Additive Approximation

\section{Introduction}

In the detailed routing phase of the design process of very large scale integrated (or VLSI) circuits the task is to interconnect certain given subsets (or nets) of

$\overline{1}$ Research partially supported by the Hungarian National Research Fund and by the National Office for Research and Technology (Grant Number OTKA 67651)

2 Email: szeszler@cs.bme.hu 
pins (or terminals) of the circuit elements that have already found their final positions on the circuit board. Since a minimum distance is to be kept between any two wires (corresponding to different nets), wires must go along the edges of a rectangular grid. However, this grid is not planar, it consists of a few planar layers (each parallel to the circuit board) and a wire can leave a layer for an adjacent one at any grid point. In some cases, certain technological constraints require that consecutive layers contain non-parallel wire segments only; if such a restriction is imposed, we speak of a Manhattan routing.

In case of the switchbox routing problem, terminals are situated on the boundaries of a rectangular circuit board. Minimizing the number of layers needed for solving a switchbox routing problem in the Manhattan model is known to be NP-hard; even very special cases of this problem are NP-hard. (For example: deciding solvability on two layers is NP-complete even if terminals are situated on two opposite boundaries only [5].) Consequently, and due to the importance of the problem, many heuristics have been proposed.

In this paper we present a linear time algorithm with a provably good performance: the number of layers used will be at most 5 greater than the optimum. Considering that the number of layers allowed by the present state of technology is typically not more than 10, this additive constant of 5 suggests that the presented construction is not intended for designing ready-to-use routing patterns for given specifications. Contrarily, we rather aim at understanding the nature of the problem and thus helping heuristics to be designed and tested. We emphasize however, that the number of layers used will stay within the realizable range of at most 10 for many realistic specifications.

\section{Basic Definitions and Preliminary Results}

The switchbox routing problem (or $S R P$ for short) is defined as follows. Assume that a $(w+2) \times(n+2)$ rectangular grid formed by $w+2$ horizontal line segments numbered from 0 to $w+1$ (called tracks) and $n+2$ vertical line segments numbered from 0 to $n+1$ (called columns) is given. Non-corner boundary points of the grid are called terminals. A net is a subset of terminals. An instance of the $S R P$ is a set $\mathcal{N}=\left\{N_{1}, \ldots, N_{t}\right\}$ of pairwise disjoint nets. The left hand side part of Figure 1 shows an $S R P$ instance with $w=n=5$; sets of terminals marked with a common number form the nets.

Terminals with a column number of 0 or $n+1$ are called western or eastern, respectively; terminals with a track number of 0 or $w+1$ are called southern or northern, respectively. $w$ and $n$ are called the width and the length of the $S R P$, respectively. The $w \times n$ grid obtained by deleting all terminals and 
corners is called the routing area. Of course, $w \leq n$ can be assumed without loss of generality; we will assume this throughout this paper. We will also assume that every net has at least two terminals. A net is called trivial if it consists of two terminals situated in a common column.

In order to define a $k$-layer solution of a $S R P$ we first replace the $w \times n$ routing area with a 3 -dimensional $w \times n \times k$ grid consisting of $k$ parallel copies (called layers) of the original routing area. In the resulting routing graph each terminal is adjacent to all the $k$ vertices of the $k$ layers corresponding to its single neighbour in the original (planar) routing area. (This is to ensure that terminals are accessible from any layer.)

A $k$-layer solution (or routing) of a $S R P$ instance $\mathcal{N}$ is a set $\mathcal{H}=$ $\left\{H_{1}, \ldots, H_{t}\right\}$ of pairwise vertex disjoint, connected subgraphs (called wires) of the routing graph such that $H_{i}$ contains all terminals of $N_{i}$ for $i=1, \ldots, t$. Edges of the wires that join adjacent vertices of two consecutive layers are called vias. Since wires can be chosen to be trees (which usually happens in every application), the $S R P$ can be regarded as a special Steiner-tree packing problem.

A solution of a $S R P$ belongs to the Manhattan model (or is simply called a Manhattan solution) if consecutive layers contain wire segments of different directions only. Thus layers with horizontal (east-west) and with vertical (north-south) wire segments alternate. In this paper we will restrict ourselves to the Manhattan model.

Denote the $i^{\text {th }}$ column of the grid by $e$ (for some $1 \leq i \leq n$ ). A nontrivial net $N$ is separated by $e$ if $N$ has a terminal with a column number less than or equal to $i$ and another terminal with a column number greater than or equal to $i$. The congestion of $e$, denoted by $c(e)$, is the number of nontrivial nets separated by $e$. The density of a $S R P$ instance, denoted by $d$, is the maximum congestion of all columns (of the routing area).

Assume that a $S R P$ instance with density $d$ is given that is solvable on $k$ layers in the Manhattan model with $h$ layers reserved for horizontal wire segments. Naturally, wires corresponding to all the $d$ nontrivial nets separated by a column $e$ with congestion $c(e)=d$ must intersect $e$ on one of these $h$ layers. At most $w$ wires can intersect $e$ on a single layer, hence $h \cdot w \geq d$ must hold. This shows (by $k \geq 2 h-1$ ) that the minimum number of layers required for solving a $S R P$ in the Manhattan model is at least $2\left\lceil\frac{d}{w}\right\rceil-1$ (and the corresponding lower bound is $\left\lceil\frac{d}{w}\right\rceil$ if the Manhattan model is not assumed).

Hambrusch [2] observed that a $S R P$ with $d=n+w$ can be constructed by letting the nets be terminal pairs, all separated by column $\left\lfloor\frac{n}{2}\right\rfloor$. This shows that the number of layers required for a $S R P$ can be as large as $2\left\lceil\frac{n}{w}\right\rceil+1$ in 
the Manhattan model (and $\left\lceil\frac{n}{w}\right\rceil+1$ otherwise) in the worst case. In particular, there is no fixed number of layers that would suffice for every $S R P$ instance.

However, there is a general upper bound for the number of layers if the ratio $\frac{n}{w}$ is fixed. This was first shown for the non-Manhattan case by Boros, Recski and Wettl [1]: they provided an upper bound of $\max \left\{18,2 \frac{n}{w}+14\right\}$. This was later improved in [4] by giving an upper bound of $2\left\lceil\frac{n}{w}\right\rceil+4$ in the Manhattan model. (Both results involve an algorithm with a linear running time.) Naturally, although the latter upper bound is only larger by 3 than the lower bound of $2\left\lceil\frac{n}{w}\right\rceil+1$ shown in the previous paragraph, the result of [4] does not give an additive approximation for the optimum since the lower bound of $2\left\lceil\frac{n}{w}\right\rceil+1$ is for the worst case only. In this paper we improve the algorithm presented in [4] to achieve an additive approximation for the optimum. (However, we do not rely on the results of [4].)

\section{Main Result}

In the previous section we have seen that the number of layers required for a $S R P$ is at least $2\left\lceil\frac{d}{w}\right\rceil-1$ in the Manhattan model, where $d$ denotes the density of the problem. This shows that the following theorem indeed guarantees a solution within an additive constant of 5 compared to the optimum.

Theorem 3.1 There is a linear time algorithm that solves every switchbox routing problem on at most $2\left\lceil\frac{d}{w}\right\rceil+4$ layers in the Manhattan model.

Proof. Assume that a $S R P$ instance $\mathcal{N}$ with density $d$ is given. We start by classifying its nets for easier reference: we say, for example, that a net is NE type if it has terminals on the northern and eastern boundaries, but none on the western and southern ones; we say that a net is $\mathrm{N}_{1} \mathrm{~W}$ type if it has one terminal on the north, some (maybe one) on the west and none elsewhere, etc. Furthermore, denote by $T_{\mathrm{W}}, T_{\mathrm{E}}, T_{\mathrm{N}}$ and $T_{\mathrm{S}}$ the set of all western, eastern, northern and southern terminals, respectively.

We can assume without loss of generality that out of NE, NW, SE and SW type nets NE nets are (one of) the greatest in number.

Denote the set of $\mathrm{S}_{1} \mathrm{~W}_{1}$ type nets by $\mathcal{N}_{0}$. Partition $\mathcal{N}_{0}$ into two subsets $\mathcal{N}_{0}^{1}$ and $\mathcal{N}_{0}^{2}$ such that $\left|\mathcal{N}_{0}^{1}\right| \leq\left|\mathcal{N}_{0}^{2}\right| \leq\left|\mathcal{N}_{0}^{1}\right|+1$. We define two further subsets of $\mathcal{N}, \mathcal{N}_{1}$ and $\mathcal{N}_{2}$. Let a nontrivial net $N \in \mathcal{N}$ belong to $\mathcal{N}_{1}$ if

- $\left|N \cap\left(T_{\mathrm{N}} \cup T_{\mathrm{S}}\right)\right| \geq 2$, but $N$ is not NE type, or

- $N$ is $\mathrm{N}_{1} \mathrm{~W}$ type, or

- $N \in \mathcal{N}_{0}^{1}$. 
Similarly, let $N \in \mathcal{N}$ belong to $\mathcal{N}_{2}$ if

- $\left|N \cap\left(T_{\mathrm{W}} \cup T_{\mathrm{E}}\right)\right| \geq 2$, but $N$ is not NE type, or

- $N$ is $\mathrm{SE}_{1}$ type, or

- $N \in \mathcal{N}_{0}^{2}$.

We assign an interval to each net of $\mathcal{N}_{1}$ and $\mathcal{N}_{2}$ : if $N \in \mathcal{N}_{1}$ then the horizontal interval $I^{H}(N)$ stretches from the column of the westernmost to the column of the easternmost terminal of $N \backslash T_{\mathrm{E}}$. Similarly, if $N \in \mathcal{N}_{2}$ then the vertical interval $I^{V}(N)$ stretches from the track of the northernmost to the track of the southernmost terminal of $N \backslash T_{\mathrm{N}}$. (In both cases, each boundary is considered when determining the endpoints of the intervals, except for the eastern boundary in case of $\mathcal{N}_{1}$ and the northern one in case of $\mathcal{N}_{2}$.) For the SRP instance of Figure 1 nets of $\mathcal{N}_{1}$ together with the assigned intervals $I^{H}(N)$ are shown in the middle part of the figure, while nets of $\mathcal{N}_{2}$ with the intervals $I^{V}(N)$ are shown in the right hand side part.
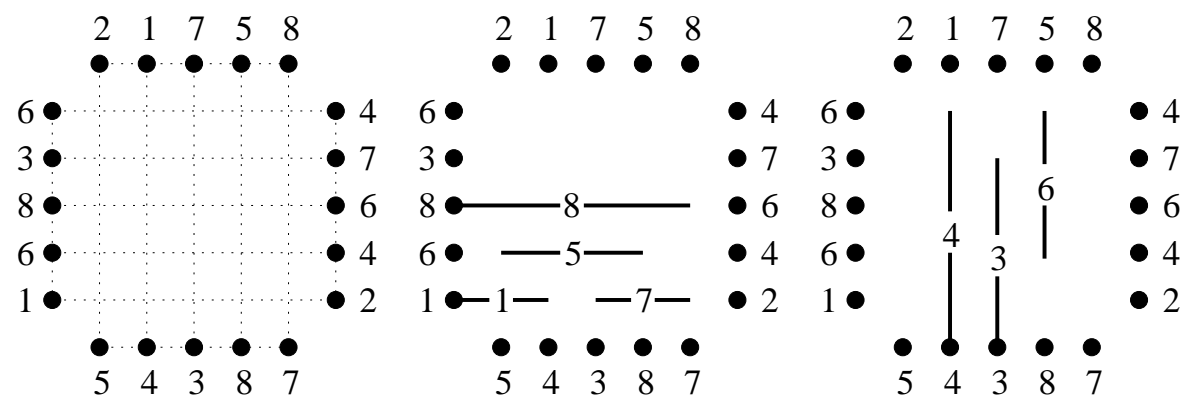

Figure 1

We claim that $\left|\mathcal{N}_{2}\right| \leq w$. Denote by $\mathcal{N}_{2}^{1}$ and $\mathcal{N}_{2}^{2}$ the first two of the three (pairwise disjoint) subsets of $\mathcal{N}$ that form $\mathcal{N}_{2}$ as listed on the top of this page (in the same order). Let $\left|\mathcal{N}_{2}^{1}\right|=x_{1},\left|\mathcal{N}_{2}^{2}\right|=x_{2},\left|\mathcal{N}_{0}\right|=y$ and let $z$ be the number of NE type nets. Since $\left|T_{\mathrm{W}} \cup T_{\mathrm{E}}\right|=2 w$, obviously $2 x_{1}+x_{2}+y+z \leq 2 w$. Because of the assumption made at the beginning of the proof, $x_{2} \leq z$ holds, thus $2 x_{1}+2 x_{2}+y \leq 2 w$ is also true. Dividing by 2 we get $x_{1}+x_{2}+\frac{1}{2} y \leq w$, which establishes the claim by $\left|\mathcal{N}_{2}\right|=x_{1}+x_{2}+\left\lceil\frac{1}{2} y\right\rceil$. (We remark that $\left|\mathcal{N}_{1}\right| \leq n$ is obviously also true with an analogous proof.)

After all this preparation we start describing the required routing. We say that a layer is a W-comb if on that layer a horizontal wire segment leads from each western terminal to the easternmost column of the routing area. The terms E-comb, N-comb and S-comb are defined analogously; however, in case of (say) $\mathrm{N}$-combs we declare that if a column contains the two terminals of a trivial net then the wire segment of that column is extended by one unit 
to the south to connect these two terminals. (We remark that the usage of comb layers simplifies the description of the construction, but obviously in most cases wire segments on the comb layers need not stretch to the opposite end of the routing area. Of course, unnecessary wire ends can be removed but we will disregard this in the sequel.)

Number the layers from bottom upwards. We declare that layer 1 is a $\mathrm{W}$-comb, layer 3 is an $\mathrm{E}$-comb, layers $4,8,12, \ldots$ are $\mathrm{N}$-combs and layers 6 , $10,14, \ldots$ are S-combs. (Since the number of layers $2\left\lceil\frac{d}{w}\right\rceil+4$ is even and at least 6 , the topmost layer is either a $\mathrm{N}$-comb or a S-comb.)

Columns of layer 2 are used for holding vertical wire segments corresponding to the intervals $I^{V}(N)$ assigned to the nets of $\mathcal{N}_{2}$. (We will also use the notation $I^{V}(N)$ to refer to the wire segment corresponding to the interval $I^{V}(N)$, this will cause no ambiguity.) We have seen that $\left|\mathcal{N}_{2}\right| \leq w$ and recall that $w \leq n$ was assumed. Consequently, each net $N \in \mathcal{N}_{2}$ can be assigned a separate column on layer 2 to hold $I^{V}(N)$. However, if $N \in \mathcal{N}_{2}$ and $N \cap T_{\mathrm{S}} \neq \emptyset$ then $I^{V}(N)$ should be placed in the column of an (arbitrary) element of $N \cap T_{\mathrm{S}}$; this way the wire segment also reaches this southern terminal of $N$, see nets 3 and 4 in Figure 1.

Consider the interval graph $G$ defined by the intervals $I^{H}(N)$ assigned to the nets of $\mathcal{N}_{1}$. (That is, $V(G)$ is the set of intervals $I^{H}(N)$ and edges of $G$ correspond to intersecting pairs of intervals. The intervals are assumed to be closed, thus two intervals are adjacent in $G$ even if they only have a common endpoint.) By definition, the clique number $\omega(G)$ of $G$ is the density $d_{1}$ of the $S R P$ defined by $\mathcal{N}_{1}$. Since $d_{1} \leq d$ is obvious from $\mathcal{N}_{1} \subseteq \mathcal{N}$, vertices of $G$ can be coloured with $d$ colours. (Here we use the well-known fact that interval graphs are perfect; this result is usually dedicated to Tibor Gallai.) Denote the colour classes by $\mathcal{I}_{1}, \ldots, \mathcal{I}_{d}$.

Tracks of layers $5,7,9,11, \ldots$ are used for holding horizontal wire segments corresponding to the intervals $I^{H}(N)$ assigned to the nets of $\mathcal{N}_{1}$. (Again, these wire segments will also be denoted by $I^{H}(N)$.) However, as opposed to the case of layer 2, we do not assign a separate track to each interval. Instead, each track is assigned to one of the colour classes $\mathcal{I}_{k}$ and the track holds wire segments corresponding to all the intervals of $\mathcal{I}_{k}$. (This is possible since intervals belonging to $\mathcal{I}_{k}$ are pairwise disjoint by the construction.) Again, if $N \in \mathcal{N}_{1}$ and $N \cap T_{\mathrm{W}} \neq \emptyset$ then the wire segments corresponding to the colour class of $I^{H}(N)$ should be placed in the track of an (arbitrary) element of $N \cap T_{\mathrm{W}}$. (This is obviously possible since no colour class can contain more than one intervals corresponding to nets having western terminals.) Since the total number of layers is $2\left\lceil\frac{d}{w}\right\rceil+4$, the number of layers $5,7,9,11, \ldots$ is $\left\lceil\frac{d}{w}\right\rceil$; 
hence the total number of tracks on these layers is at least $d$ which shows that each colour class $\mathcal{I}_{k}$ can be assigned an appropriate track.

The routing is now completed by introducing vias between consecutive layers appropriately. For every $N \in \mathcal{N}_{2}$ the vertical wire segment $I^{V}(N)$ on layer 2 can be connected to the horizontal wire segments of layers 1 and 3 coming from the terminals of $N \cap\left(T_{\mathrm{W}} \cup T_{\mathrm{E}}\right)$. Similarly, for every $N \in \mathcal{N}_{1}$ the horizontal wire segment $I^{H}(N)$ can be connected to the vertical wire segments coming from the terminals of $N \cap\left(T_{\mathrm{N}} \cup T_{\mathrm{S}}\right)$ in the layers below and above the layer of $I^{H}(N)$. Finally, we introduce vias between layers 3 and 4 suitably: for every $s \in T_{\mathrm{N}}$ and $t \in T_{\mathrm{E}}$ if $s$ and $t$ belong to the same net then the vertical wire segment coming from $s$ on layer 4 and the horizontal wire segment coming from $t$ on layer 3 can be connected through an appropriate via. (Evidently, it is not necessary to take advantage of all the possibilities to introduce the above mentioned vias between layers 3 and 4 . Moreover, it is not only unnecessary, but for certain $S R P$ instances the number of - mainly needless - vias could become so high that the algorithm would not be linear any more. We disregard this detail in the sequel with mentioning that for each net $N$ the number of vias needed between layers 3 and 4 is at most $\left|N \cap\left(T_{\mathrm{N}} \cup T_{\mathrm{E}}\right)\right|$.)

We claim that the above described routing is good. Choose a net $N \in \mathcal{N}$ and two terminals $s, t \in N$ arbitrarily. It is sufficient to show that $s$ and $t$ are connected by the routing. If $N$ is trivial then $s$ and $t$ are joined in the corresponding column of a $\mathrm{N}$-comb. If $N$ is NE type then the routing of $N$ is completely solved by the vias between layers 3 and 4 . So we can assume that $N$ is neither trivial nor NE type; it is easy to check that this implies $N \in \mathcal{N}_{1} \cup \mathcal{N}_{2}$. If $\{s, t\} \subseteq T_{\mathrm{W}} \cup T_{\mathrm{E}}$ then $N \in \mathcal{N}_{2}$ and hence $s$ and $t$ are connected through $I^{V}(N)$. Similarly, if $\{s, t\} \subseteq T_{\mathrm{N}} \cup T_{\mathrm{S}}$ then $N \in \mathcal{N}_{1}$ and $s$ and $t$ are connected through $I^{H}(N)$. So we can assume that $s$ and $t$ belong to two adjacent boundaries.

Assume first that $s \in T_{\mathrm{W}}$ and $t \in T_{\mathrm{N}}$. If $N \cap T_{\mathrm{E}} \neq \emptyset$ and $u \in N \cap T_{\mathrm{E}}$ then $N \in \mathcal{N}_{2}, u$ and $s$ are connected through $I^{V}(N), u$ and $t$ are connected through the vias between layers 3 and 4 and hence $s$ and $t$ are also connected via $u$. If, on the other hand, $N \cap T_{\mathrm{E}}=\emptyset$ then $N \in \mathcal{N}_{1}$ by definition and hence $t$ is connected to a terminal $s^{\prime} \in N \cap T_{\mathrm{W}}$ through $I^{H}(N)$. If $s^{\prime} \neq s$ then $N \in \mathcal{N}_{2}$ is also true, $s$ and $s^{\prime}$ are connected through $I^{V}(N)$ so $s$ and $t$ are connected via $s^{\prime}$. The case $s \in T_{\mathrm{E}}, t \in T_{\mathrm{S}}$ is analogous. If $s \in T_{\mathrm{W}}$ and $t \in T_{\mathrm{S}}$ then either $N \in \mathcal{N}_{1}$ and $t$ is connected to a terminal $s^{\prime} \in N \cap T_{\mathrm{W}}$ through $I^{H}(N)$ or $N \in \mathcal{N}_{2}$ and $s$ is connected to a terminal $t^{\prime} \in N \cap T_{\mathrm{S}}$ through $I^{V}(N)$; in the first case $s \neq s^{\prime}$ implies $N \in \mathcal{N}_{2}$ and $s$ and $s^{\prime}$ are connected through $I^{V}(N)$ while in the second case $t \neq t^{\prime}$ implies $N \in \mathcal{N}_{1}$ and $t$ and $t^{\prime}$ 
are connected through $I^{H}(N)$. Finally, if $s \in T_{\mathrm{E}}$ and $t \in T_{\mathrm{N}}$ then $s$ and $t$ are simply connected by the vias between layers 3 and 4 .

We omit the proof that the above construction can be realized by a linear time algorithm for space reasons. We restrict ourselves to mentioning that the only nontrivial step is the colouring of the interval graph $G$ defined above; the reader is referred to [3] for the details.

\section{Concluding Remarks}

We remark that the above proof gives a slightly better upper bound on the number of layers than the one claimed by Theorem 3.1: $2\left\lceil\frac{d_{1}}{w}\right\rceil+4$, where $d_{1}$ is the density of the $S R P \mathcal{N}_{1}$ (defined in the proof). Observe that the maximum value of $d_{1}$ is $n$ (since $\left|\mathcal{N}_{1}\right| \leq n$ was mentioned in the proof) while the value of $d$ can be as large as $n+w$ (as mentioned in the Introduction). This implies that the above upper bound of $2\left\lceil\frac{d_{1}}{w}\right\rceil+4$ can indeed be less than the one claimed in Theorem 3.1. Furthermore, it also shows that the construction of the above proof generalizes the result of [4] mentioned in the Introduction by $2\left\lceil\frac{d_{1}}{w}\right\rceil+4 \leq 2\left\lceil\frac{n}{w}\right\rceil+4$.

It is also worth mentioning that although the additive constant guaranteed by Theorem 3.1 is 5 in general, however, in certain special cases this can be improved. For example, if $d_{1} \leq w$ holds (which is definitely true in the $n=w$ case) then the construction guarantees a routing on 6 layers; since a 1-layer solution is clearly impossible (except for trivial cases), the additive constant of approximation is 4 in this case.

\section{References}

[1] Boros, E., A. Recski and F. Wettl, Unconstrained multilayer switchbox routing, Annals of Operations Research 58 (1995), 481-491.

[2] Hambrusch, S. E., Channel routing in overlap models, IEEE Trans. ComputerAided Design of Integrated Circ. Syst. CAD-4 (1985), 23-30.

[3] Szeszlér, D., Combinatorial Algorithms in VLSI Routing, PhD Dissertation, 2005. http://www.cs.bme.hu/ szeszler/szeszler_dissertation.pdf

[4] Szeszlér, D., Switchbox routing in the multilayer Manhattan model, Annales Univ. Sci. Budapest. Eötvös Sect. Math. 40, (1997), 155-164.

[5] Szymanski, T. G., Dogleg channel routing is NP-complete, IEEE Trans. Computer-Aided Design of Integrated Circ. Syst. CAD-4 (1985), 31-41. 